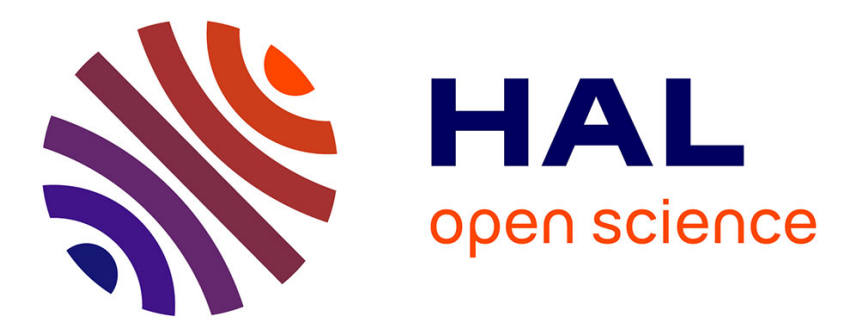

\title{
Putative orthologues of genetically identified Drosophila melanogaster chitin producing and organising genes in Apis mellifera
}

\author{
Yiwen Wang, Richard Odemer, Peter Rosenkranz, Bernard Moussian
}

\section{- To cite this version:}

Yiwen Wang, Richard Odemer, Peter Rosenkranz, Bernard Moussian. Putative orthologues of genetically identified Drosophila melanogaster chitin producing and organising genes in Apis mellifera . Apidologie, 2014, 45 (6), pp.733-747. 10.1007/s13592-014-0292-3 . hal-01234775

HAL Id: hal-01234775

https://hal.science/hal-01234775

Submitted on 27 Nov 2015

HAL is a multi-disciplinary open access archive for the deposit and dissemination of scientific research documents, whether they are published or not. The documents may come from teaching and research institutions in France or abroad, or from public or private research centers.
L'archive ouverte pluridisciplinaire HAL, est destinée au dépôt et à la diffusion de documents scientifiques de niveau recherche, publiés ou non, émanant des établissements d'enseignement et de recherche français ou étrangers, des laboratoires publics ou privés. 


\title{
Putative orthologues of genetically identified Drosophila melanogaster chitin producing and organising genes in Apis mellifera
}

\author{
Yiwen $\mathrm{WANG}^{1}$, Richard Odemer ${ }^{2}$, Peter Rosenkranz ${ }^{2}$, Bernard Moussian ${ }^{1}$ \\ ${ }^{1}$ Animal Genetics, Interfaculty Institute for Cell Biology, Eberhard-Karls University of Tübingen, Auf der \\ Morgenstelle 8, 72076, Tübingen, Germany \\ ${ }^{2}$ Apicultural State Institute, University of Hohenheim, August-von-Hartmannstr. 13, 70599, Stuttgart, Germany
}

Received 1 August 2013 - Revised 17 March 2014 - Accepted 30 April 2014

\begin{abstract}
The cuticle is a versatile coat allowing insects to survive in a variety of ecological situations. The polysaccharide chitin is a major cuticle component defining cuticle function. The key players of chitin synthesis and organisation, including chitin synthase-1 (CS-1), the chitin deacetylases vermiform (Verm) and serpentine (Serp), the extracellular obstructor-A (Obst-A) protein, the membrane-bound Knickkopf (Knk) and retroactive (Rtv) proteins, the cytoplasmic mummy (Mmy) protein and the transcription factor grainyhead (Grh) have been characterised genetically in the fruit fly Drosophila melanogaster. Here, we report on the identification of putative orthologues of these factors in Apis mellifera and on their expression at different developmental stages. Overall, differences in gene organisation and expression pattern between these two species may compile different cuticle compositions that reflect their life style and ecology. Our data will serve to elucidate the honeybee-specific mechanisms of cuticle formation.
\end{abstract}

cuticle / chitin / chitin synthase / chitin organisation

\section{INTRODUCTION}

Insects are covered by a cuticle that protects them against dehydration and pathogen entry and allows locomotion. In addition, the cuticle stabilises the body as well as internal organs such as the foregut, the hindgut and the tracheae. It is a stratified extracellular matrix that is produced by the underlying epithelial cells at their apical site (Moussian 2010, 2013). Stratification is the result of ordered sorting and

Electronic supplementary material The online version of this article (doi:10.1007/s13592-014-0292-3) contains supplementary material, which is available to authorized users.

Corresponding author: B. Moussian, bernard.moussian@uni-tuebingen.de Manuscript editor: Marina Meixner arrangement of distinct components. The outermost layer is the envelope, which mainly consists of a species-specific mixture of hydrocarbons and wax esters. Underneath the envelope lies the epicuticle that is composed of yet unidentified proteins and probably lipids. Bundles of the polysaccharide chitin associate with chitin-binding proteins and form horizontal sheets (called laminae) that are stacked constituting the innermost cuticle layer, the procuticle. While the histology of the cuticle of various arthropods has been meticulously described in the last decades (Locke 2001), the molecular mechanisms of cuticle differentiation are being investigated only recently in detail. Genetic approaches using the fruit fly Drosophila melanogaster have advanced our understating especially in the field of chitin production and organisation. Subsequently, several factors iden- 
tified and characterised in D. melanogaster have been studied in the red flour beetle Tribolium castaneum by RNA interference, largely confirming results obtained with $D$. melanogaster and occasionally adding new notions to their function. For instance, Knickkopf (Knk), a membrane-bound chitin organising factor in D. melanogaster (Moussian et al 2006), is additionally needed to protect chitin from chitinase-driven degradation in $T$. castaneum (Chaudhari et al 2011). Hence, despite the equivalence of chitin synthesising and organising factors in D. melanogaster and T. castaneum, functional differences exist. The significance of these differences remains to be investigated.

In order to deepen our understanding in variability of the molecular mechanisms deployed during insect cuticle formation, we sought to identify those chitin producing and organising factors in the honeybee Apis mellifera that had been previously demonstrated to be essential in $D$. melanogaster in genetic experiments. These factors that supposedly constitute the core factors of chitin synthesis and organisation are krotzkopf verkehrt/chitin synthase-1 (Kkv/CS-1), the chitin monomer (GlcNAc) producing enzyme mummy (Mmy, UDP-GlcNAc pyrophosphorylase), the membrane-proteins Knickkopf (Knk) and retroactive (Rtv) and the extracellular proteins obstructor-A (Obst-A), serpentine (Serp) and vermiform (Verm), two chitin deacetylases (Gangishetti et al 2012; Luschnig et al 2006; Moussian et al 2005a, b, 2006; Petkau et al 2012; Tonning et al 2006). Mutations in the respective genes are lethal and cause chitin deficiency $(C S-1 / k k v, m m y)$ or chitin disorganisation ( $k n k$, obst-A, rtv, verm, serp). In addition, we identified the $A$. mellifera grainyhead (Grh) transcription factor that in $D$. melanogaster has been shown to control the transcription of several chitin producing and organising factors such as knk (Bray and Kafatos 1991; Gangishetti et al 2012; Pare et al 2012). In order to evaluate especially the presence of newly discovered exons and to study expression dynamics, we also monitored the expression of these genes at different developmental stages.

\section{MATERIALS AND METHODS}

\subsection{Animal husbandry}

Honeybee (A. mellifera) larvae and pupae were obtained from the Apicultural State Institute at the University of Hohenheim, Germany.

\subsection{Identification of putative chitin producing and organising factors in A. mellifera}

In order to identify putative orthologues of $D$. melanogaster genetically characterised chitin producing and organising factors in A. mellifera, we established and followed a five-step protocol. First, we searched the bee genome for respective loci with the coding sequence of $D$. melanogaster factors using the tBLASTx software at BeeBase (hymenopteragenome.org/beebase) (Munoz-Torres et al 2011). In general, searches yielded fragments coding for protein sequences highly homologous to the D. melanogaster sequence. Second, the exons of the annotated sequence encoding the closest homologue were assembled to a continuous coding sequence that was translated to a protein sequence using the SerialCloner 2-6-1 software. Third, the conceptual $A$. mellifera protein sequence was subsequently used to search the $D$. melanogaster protein sequence database at FlyBase for homologous sequences by BLASTp. Without any exception, this search confirmed the closest similarity between the respective initial $D$. melanogaster and A. mellifera sequences. Usually, the protein sequences were colinear. Co-linearity of $D$. melanogaster and $A$. mellifera protein sequences is considered as an indication that we had identified all exons correctly. In parallel, our deduced honeybee protein sequences were aligned with A. mellifera annotated protein sequences at the NCBI gene bank by BLASTp. Surprisingly, there were substantial differences in several cases; these differences are dealt within the "Results" section. Ambiguous exons were tested by qualitative PCR whether they are part of respective transcripts or not. The fourth step of our protocol was to use the protein organisation retrieved from the genomes of the honeybee-related species Bombus impatiens, Nasonia vitripennis and Camponotus 
floridanus to corroborate exon composition of the $A$. mellifera gene. Fifth, expression of genes in $A$. mellifera was recorded at the first (L1) and fourth (L4) instar larval and pink-eyed pupal stages.

\subsection{Sequence analyses}

Protein domains were initially identified during sequence comparison using the BLASTp software at the National Centre for Biotechnology Information (NCBI). Subsequently, they were studied at the Conserved Domain (NCBI) and Pfam (pfam.sanger.ac.uk/) databases. Putative signal peptides were detected with the PSORT software (psort.hgc.jp). Glycosylphosphatidylinositol (GPI) anchors were predicted using the big-PI Predictor software (mendel.imp.ac.at/gpi/gpi_server.html). Transmembrane domains were predicted using the TMPred software at embnet.vital-it.ch/software/ TMPRED_form.html. Multiple sequences were aligned using the ClustalO software at www.uniprot.org/align/. For phylogenetic analyses, neighbour-joining trees were constructed using the MEGA 5.2.2. software that allows testing correctness of the relationships by the bootstrap test $(1,000$ calculations, method: $p$-distance).

To search for possible exons in the $5^{\prime}$ region of GB46725 (coding for the putative grainyhead orthologue), the respective region was scanned manually and by using the software Fgenesh+ (www.softberry.com) and Genscan (genes.mit.edu/ GENSCAN.html).

\subsection{Molecular biology}

Total RNA was extracted from 50 first (L1) and two fourth instar larvae (L4) and two pink-eyed pupae using the Qiagen RNEasy kit (Hilden, Germany). For each stage, two independent RNA extractions were carried out. PCR experiments with primers recognising genomic DNA but not complementary DNA (cDNA) proved the absence of genomic DNA (data not shown). Total RNA served as template to produce cDNA by oligodT-primed reverse transcription using the Roche Transcriptor First-Strand cDNA Synthesis kit (Mannheim, Germany). Qualitative PCR was performed on a LightCycler Nano from Roche using the Roche FastStart SYBR Green
Master kit (Mannheim, Germany). In our qualitative PCR experiments, we followed the guidelines proposed by Bustin et al (2009). Amplification of tubulin (GB44134, most similar sequence to D. melanogaster tub50) transcripts was performed to verify that the dynamic range of amplification was linear at three template concentrations ( -10 -fold, 1 -fold, +10 -fold). The primers used for transcript amplification were designed with the Primer3 software (biotools.umassmed.edu/bioapps/primer3_www.cgi) and produced by Sigma-Aldrich; they are listed in Supplementary Table 1. Data based on ddCq values and amplification efficiencies were analysed with the respective Roche LightCycler software and Microsoft Excel; efficiency values for each pair of primers are indicated in Supplementary Table 1. As a control, primers to amplify tubulin were used in each experiment.

To analyse the presence of the exon-pairs XIV/XV and XVIII/XIX in chitin-synthase coding transcripts, we first amplified the sequences between exons XIII to XVII and XVI to XX from L4 larval cDNA (generated using the Sigma-Aldrich Enhanced Avian RT First Strand Synthesis Kit) using appropriate primers (Supplementary Table 1). Second, we used the respective amplicons to amplify and determine sequences between exons XIII and XIV or XV and exons XVI and XVIII or XIX using appropriate specific primers (Supplementary Table 1).

\section{RESULTS}

\subsection{The chitin synthase (CS-1/Kkv)}

Chitin is synthesised by the membraneinserted chitin synthase. Mutations in the $D$. melanogaster chitin synthase coding gene krotzkopf verkehrt $(k k v)$ are lethal and cause a chitin-deficient collapsed cuticle (Moussian et al 2005a). The $k k v$ locus codes for two enzyme isoforms with 1,615 amino acids derived from two alternative transcripts harbouring either exon VIIa or VIIb (Irion 2012). The $k k v$-similar A. mellifera GB49845 locus is annotated to contain 23 exons coding for a conceptual protein with 1,783 amino acids (Figure 1). Sequence comparison suggests that duplicated stretches of sequences encoded by alternative 
exons account for the non-linearity and overlength of the $A$. mellifera sequence. For instance, exons XIV and XV (102 aa) both contain the WGTRE motif that has been proposed to be essential for chitin extrusion (Merzendorfer 2006). This motif is at the beginning of a long stretch of a coiled-coil forming sequence (residues 1,121 to 1,224 , in D. melanogaster: 1,071 to 1,173; Fig. S1). Other insect chitin synthases contain only one WGTRE motif arguing that exons XIV and XV are mutually exclusive. By qualitative PCR (Table II) and sequence determination (data not shown), we confirmed that these exons are not present on the same transcript, i.e. they are most probably alternatively spliced in larvae and pupae.

We next asked whether the duplication of the WGTRE coding exon is specific to A. mellifera or Hymenoptera. To answer this question, we characterised the genes coding for the epidermal chitin synthase in the Hymenoptera $B$. impatiens (Apidae, i.e. closely related to $A$. mellifera, (Johnson et al 2013)), C. floridanus (Formicidae, i.e. intermediate relative of $A$. mellifera) and $N$. vitripennis (Pteromalidae, i.e. distantly related to A. mellifera). D. melanogaster served as an out-group (Table I). The duplicated exon is present in B. impatiens but not in $C$. floridanus, $N$. vitripennis or $D$. melanogaster. This finding indicates that the exon was duplicated after the separation of the ancestor of $A$. mellifera and $B$. impatiens from the common ancestor of ants, small parasitoid wasps and bees.

Another pair of exons that like their respective exons in other insects (Arakane et al 2004, 2005; Hogenkamp et al 2005; Rezende et al 2008) may be alternatively spliced is exons XVIII (180 bps) and XIX (174 bps). Both exons are predicted to code for transmembrane domains, which are highly similar (66\% identical bases coding for $80 \%$ similar and $69 \%$ identical amino acids, respectively). However, our qualitative PCR data using two independent pairs of primers suggest that in A. mellifera, exons XVIII and XIX may be present in the same transcript in larvae and pupae (Table II). If both exons are present in the same transcript, the C-terminus of the resulting protein would be cytoplasmic instead of extracellular as compared to the situation in D. melanogaster. To scrutinise whether transcripts with alternative exons XVIII and XIX exist, we performed RTPCR experiments and determined the sequence of the resulting amplicons. Indeed, the vast majority of amplicons harboured only one of the exons (data not shown).

In summary, the conceptual $A$. mellifera chitin synthase with only one WGTRE motif and excluding the transmembrane domain encoded by exon XIX has 1,623 residues and is largely collinear with the respective $D$. melanogaster protein. Over 1,599 residues, they are $80.2 \%$ similar and $66.4 \%$ identical to each other.

The major non-aligning region is present at the N-terminus, which is variable in chitin synthases in all insects (Hogenkamp et al 2005; Merzendorfer 2006). The predicted NCBI A. mellifera chitin synthase protein has 1,632 amino acids that are, except from ten unique $\mathrm{N}$ terminal amino acids, identical to the conceptual BeeBase sequence (Fig. S1). In qualitative PCR experiments, we could not detect the $5^{\prime}$ region of the A. mellifera chitin synthase transcript deposited at the NCBI site, whereas the BeeBase $5^{\prime}$ sequence of the chitin synthase transcript could be amplified (Table II).

\subsection{GlcNAc pyrophosphorylase}

The monomer of chitin, $\mathrm{N}$-acetylglucosamine (GlcNAc), which is also a major component of $\mathrm{N}$-glycans, is produced by the Leloir pathway (Moussian 2008). The last enzyme of this pathway catalysing the formation of UDPGlcNAc is the cytoplasmic GlcNAc pyrophosphorylase, named mummy (Mmy) in $D$. melanogaster (Araujo et al 2005; Schimmelpfeng et al 2006; Tonning et al 2006). D. melanogaster larvae homozygous for loss-of-function mmy alleles have, among others, a strongly reduced cuticle and die before hatching. The mmy gene codes for two isoforms with 520 (A) and 483 (B) amino acids, isoform A having a longer N-terminus (Figure 2). The A. mellifera locus $G B 44897$ codes for a protein with 469 amino acids encompassing the GlcNAc pyrophosphorylase signature from residue 84 to residue 406 (PF01704). It is collinear 
A

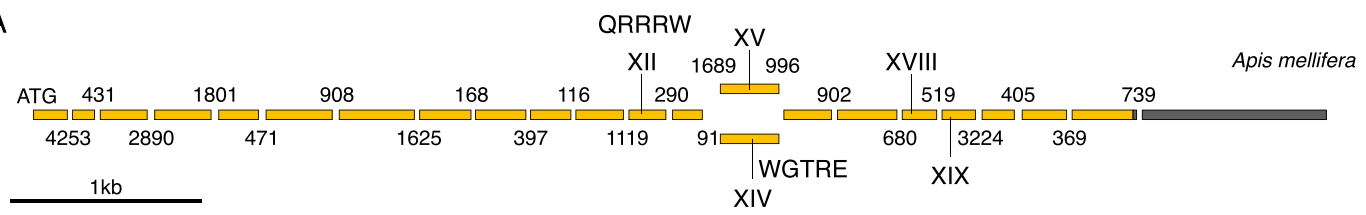

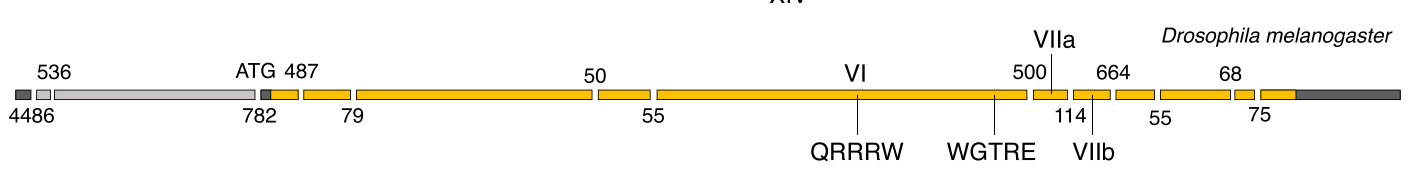

B Dmel-AC DKDRIRHRKRWSQVMYMYYLLGHRLMELPISVDRKDAIAENTYLLTLDGDIDFKPNAVTL 60 Amel-AC DKSKIRHRKRWSQVMYMYYLLGHRLMELPISVDRKEVIAENTYLLTLDGDIDFQPAAVKL 60 $* * .: * * * * * * * * * * * * * * * * * * * * * * * * * * * * * * *: . * * * * * * * * * * * * * * * *::^{*} * * . *$

Dmel-AC LVDLMKKNKNLGAACGRIHPVGSGPMVWYOLFEYAIGHWLQKATEHMIGCVLCSPGCFSL 120 Amel-AC LVDLMKKNKNLGAACGRIHPVGSGPMVWYQMFEYAIGHWLQKATEHMIGCVLCSPGCFSL 120 $* * * * * * * * * * * * * * * * * * * * * * * * * * * * * *: * * * * * * * * * * * * * * * * * * * * * * * * * * * * *$

Dmel-AC FRGKALMDDNVMKKYTTRSDEARHYVQYDQGEDRWLCTLLLQRGYRVEYSAASDAYTHCP 180

Amel-AC FRGKALMDDNVMKKYTTRSDEARHYVQYDQGEDRWLCTLLLQRGYRVEYSAASDAYTHAP 180

Dmel-AC EGFNEFYNQRRRWVPSTIAN 200

Amel-AC EGFNEFYNQRRRWVPSTIAN 200 $* * * * * * * * * * * * * * * * * * * *$

C Dmel LFVAQVLSTAYALIMMAVIVGTALQLGEDGIGSPSAIFLISMVGSFFIAACLHPQEFWCI 60 AmelXIV LLCAQILSTGYAMIMMAVIVGTALQLGEDGIGSPSAIFLIALSGSFFIAACLHPQEFWCI 60 AmelXV VFVAAIISTLYGMIMVAVIVGTAINIVODGILSPSAMFLMLLMSOIVITAILHTEEIFCI 60 $:: *:: * * * .: * *: * * * * * * *:::: * * * * * * *: * *:: \ldots:$ : $^{*} * * * .:^{*}::^{* *}$

Dmel TCGLIYLLSIPSMYLLLILYSIINLNVVSWGTREV------- 95

AmelXIV VPGIIYLLSIPSMYLLLILYSIINLNVVSWGTREVQVKKTKK 102

AmelXV IYSVIYFITIPSMYVLLIIYSVCNLNDITWGTREIKTRKTAA 102 : :**:::*****:***:**: ***:::****:

Figure 1. The chitin synthase locus. a The D. melanogaster kkv gene has 12 exons (dark grey boxes represent non-coding sequences; orange boxes represent coding sequences), whereas the A. mellifera chitin synthase gene has 23 exons. In D. melanogaster, exons I and II are separated by two genes, CG14668 and CR31593 (light grey). The chitin synthase signature (glycosyltransferase like family 2: PF13641) is encoded by four exons (exons IX-XII), whereas in D. melanogaster, this domain is encoded by only one exon (exon VI). b The respective amino acid sequences (active centre, $A C$ ) in $D$. melanogaster (Dmel) and A. mellifera (Amel) share $95.5 \%$ identical and $97.5 \%$ similar residues. c In contrast to the situation in D. melanogaster (Dmel), the sequence coding for the WGTRE motif is separated from the signature coding exons by an interjacent exon. The WGTRE coding exon is duplicated in A. mellifera (Amel). The exon XIV encoded protein sequence displays a higher identity $(88.4 \%)$ and similarity $(93.7 \%)$ to the respective D. melanogaster sequence than does the exon XV encoded sequence (53.7 and $77.9 \%$ ). A full alignment of epidermal chitin synthases in $D$. melanogaster, A. mellifera and related insects is shown in Supplementary Figure 1. The sizes of introns are indicated between the exon boxes. Symbols underneath the alignment represent conserved (asterisk) or similar amino acids (colon strong similar properties; period weak similar properties) according to the Clustal Omega guidelines at http://www.ebi.ac.uk/Tools/msa/clustalo/help/.

with the $D$. melanogaster isoform A and shows $53.8 \%$ identity and $73.8 \%$ similarity to it over the entire length of the protein and $58.3 \%$ identity and $76.9 \%$ similarity over the PF01704 domain (Fig. S2). Since GB44897 is a housekeeping gene, we did not monitor its expression at different developmental stages.

\subsection{The chitin deacetylases serpentine and vermiform}

Chitin modification by the two secreted chitin deacetylases serpentine (Serp) and vermiform (Verm) have been reported to be essential for chitin organisation (Gangishetti et al 2012; 


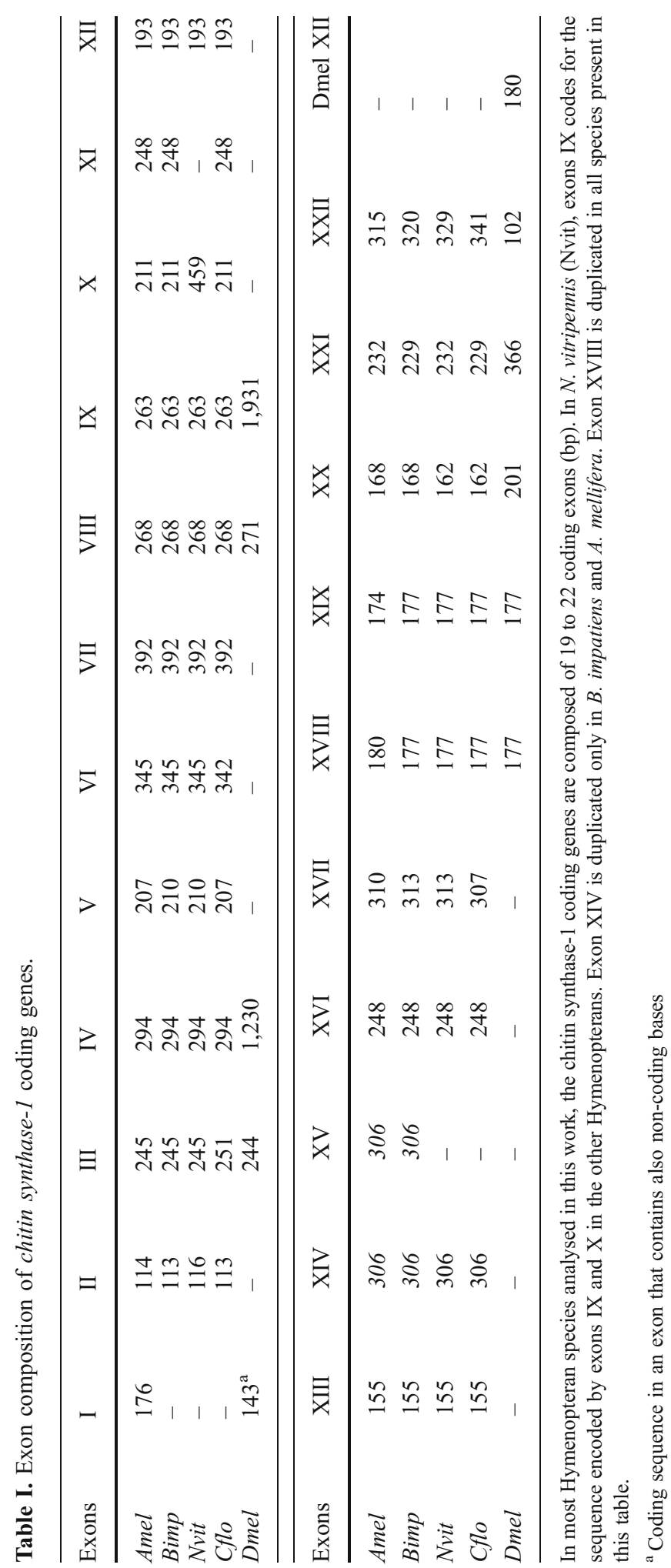


Table II. Expression profiles of chitin producing and organising factors in A. mellifera.

\begin{tabular}{llcl}
\hline Orthologue & Probe & First instar larvae & Pink-eyed pupae \\
\hline & GB49845 exons VIII-IX & + & + \\
& 5' GB49845 from BeeBase & + & n.d. \\
& 5' GB49845 from NCBI & - & n.d. \\
& GB49845 exon XIV & + & + \\
$k k v$ & GB49845 exons XIV-XV & - & - \\
& GB49845 exon XV & + & + \\
& GB49845 exon XVIII & + & + \\
& GB49845 exons XVIII-XIX a & + & + \\
& GB49845 exon XIX & + & + \\
serp & GB49845 exons XVIII-XIX b & + & + \\
verm & GB45151 & + & + \\
$k n k$ & GB45152 & - & + \\
rtv & GB50061 & + & + \\
obst- $A$ & GB49764 & + & + \\
grh & GB50636 & + & + \\
& GB46725 exons V-VI & n.d. & + \\
\hline
\end{tabular}

The expression of genes (probe) coding for chitin producing and organising factors in A. mellifera was recorded in first instar larvae and pink-eyed pupae by qualitative PCR using the primers listed in Supplementary Table 1 . The putative $D$. melanogaster orthologues are indicated (orthologue). Expression is marked by a + , non-expression is represented by a -. Expressions of the 5' end of GB49845 (5' GB49845 Beebase and 5' GB49845 NCBI) and of exons V and VI of GB46725 (grh) were not determined in pupae (n.d.)

Luschnig et al 2006). The A. mellifera GB45151 locus codes for a protein with 532 amino acids, which is $89.9 \%$ identical and $95 \%$ similar to the D. melanogaster Serp protein (isoform B: $541 \mathrm{aa})$ over almost the entire protein sequence (516 residues), excluding the $\mathrm{N}$-terminal signal peptide (Figure 3). Over 511 residues, the GB45151 protein is $61.4 \%$ identical and $77.5 \%$ similar to Verm (549 aa). The neighbouring locus GB45152 encodes another chitin deacetylase with 549 amino acids that over 519 residues are $84 \%$ identical and $91.3 \%$ similar to Verm and over 545 residues $56.1 \%$ identical and $73.4 \%$ similar to Serp.

Aside from the sequences in the A. mellifera genome, we identified the respective loci in the Hymenoptera $B$. impatiens, $C$. floridanus and $N$. vitripennis. In general, exon composition of the serp- and verm-like chitin deacetylase coding genes is conserved among the four Hymenoptera representatives. In phylogenetic analyses, the respective proteins segregate into two distinct clades, each branch harbouring one sequence from each species tested (Fig. S3).

As in $A$. mellifera, the respective chitin deacetlyase coding genes serp and verm in $D$. melanogaster are arranged in tandem in the genome (Luschnig et al 2006). We found that the cognate loci are also arranged in tandem in the genomes of $B$. impatiens and $N$. vitripennis (Figure 4). Incomplete assembly of the $C$. floridanus genome does not allow any conclusion on gene arrangement in this species. Nevertheless, the tandem arrangement suggests an early duplication of a chitin deacetylasecoding gene in the insect linage.

The putative A. mellifera Serp (GB45151) and Verm (GB45152) orthologues are differen- 


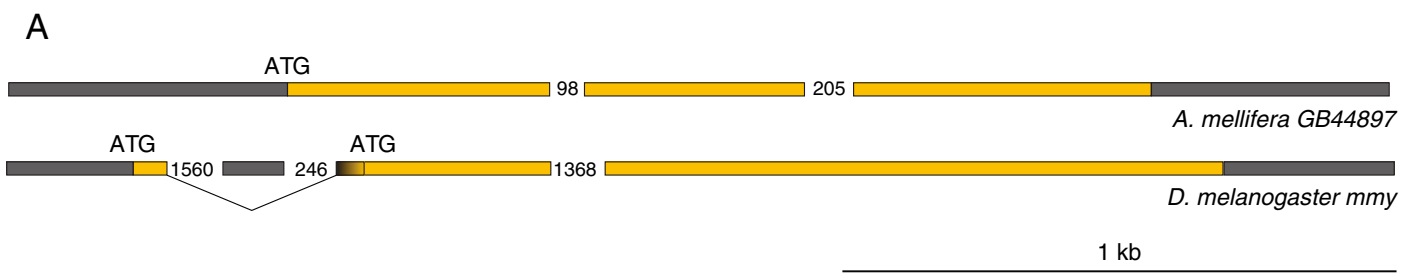

Figure 2. The mmy locus. The D. melanogaster mmy locus has two alternative start codons $(A T G)$ and three coding exons (orange boxes; grey boxes represent non-coding sequences). The A. mellifera gene $G B 44897$ has only one predicted start codon and three coding exons. RNA sequencing data at BeeBase do not contradict the comparably simple composition of the GB44897 locus. A full alignment of Mmy and Mmy-like proteins in $D$. melanogaster, A. mellifera and related insects is shown in Supplementary Figure 2. The sizes of introns are indicated between the exon boxes.

tially expressed (Table II). While the GB45151 transcript is detected at larval stages, the GB45152 transcript is pupal specific.

\subsection{The chitin organising factor Knickkopf}

The D. melanogaster Knickkopf protein is a membrane-anchored protein that is needed for correct chitin organisation during larval cuticle formation (Moussian et al 2006). In the flour beetle $T$. castaneum, Knk additionally protects chitin against degradation by chitinases before moulting (Chaudhari et al 2011). The $A$. mellifera GB50061 locus consisting of 13 exons codes for a protein with 686 amino acids that displays $57.6 \%$ identity and $72.4 \%$ similarity over its entire length to the D. melanogaster Knk protein (689 aa, Figure 5 and S4). The $D$. melanogaster Knk protein has an N-terminal signal peptide, a tandem of DM13 domains, a central DOMON domain and a C-terminal signature allowing the addition of a GPI anchor. GB50061 is predicted to have an N-terminal signal peptide that is cleaved at Gly30. A tandem of DM13 domains and the DOMON domain is present in GB50061, as well. In addition, the $\mathrm{C}$-terminus possesses the signature for GPI anchor attachment. Taken together, the overall architectures of GB50061 and Knk are identical. GB50061 shows significant albeit weaker similarity to two additional $D$. melanogaster proteins, CG34355 (37.9\% identity/56.4\% similarity) and CG43161 (38.7\% identity $/ 56.1 \%$ similarity). The transcript of GB50061 is detected at the larval and pupal stages (Table II).

\subsection{The chitin organising factor retroactive}

The D. melanogaster Retroactive (Rtv) protein is a membrane-bound Ly6-type protein that assists chitin organisation during embryogenesis (Moussian et al 2005b, 2006). In T. castaneum, the Rtv assists trafficking of Knk to the apical plasma membrane and the extracellular space (Chaudhari et al 2013). The A. mellifera GB49764 locus contains three exons coding for a protein with 146 amino acids, which is highly similar to the D. melanogaster Rtv protein (Figure 4). Over the length of 136 amino acids, this protein is $52.2 \%$ identical and $66.9 \%$ similar to the D. melanogaster Rtv protein (151 aa; Fig. S5). GB49764 has an N-terminal signal peptide and a Cterminal signature for GPI modification (omega-site N122). Structure-based sequence homology searches using the HHpred software (Soding et al 2005) confirm that the sequence between the N-terminal signal peptide and the C-terminal transmembrane domain adopts a Ly6-like folding (data not shown), which in some cases has been 
A

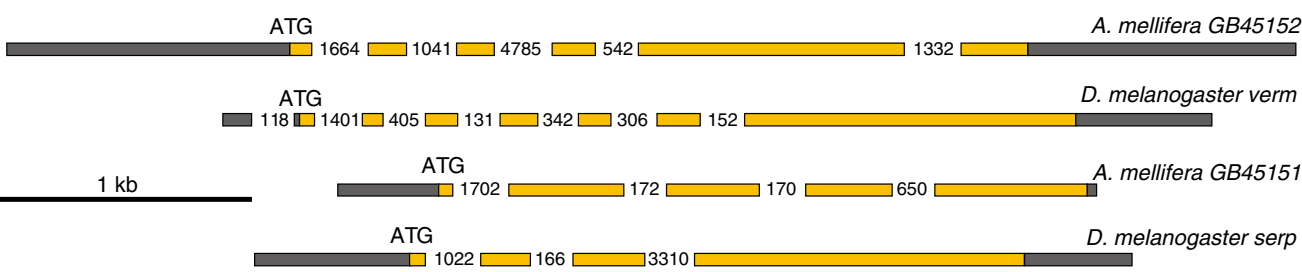

B

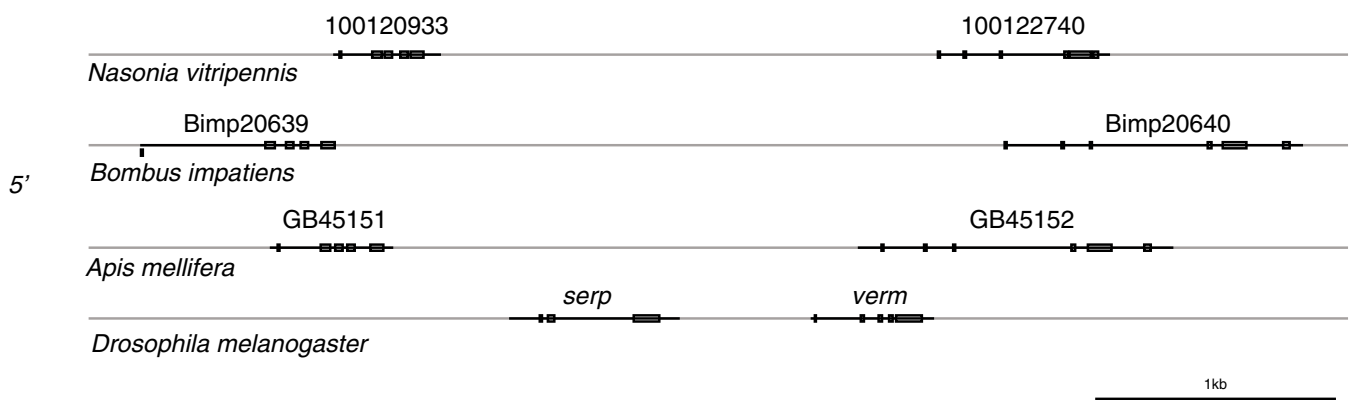

Figure 3. The serp and verm loci. a The D. melanogaster genes serp and verm code for chitin deacetylases. The A. mellifera GB45151 locus coding for the Serp orthologue has five exons (grey boxes represent noncoding sequences; orange boxes represent coding sequences). The A. mellifera GB45152 locus that encodes the Verm orthologue has six exons. According to Flybase, exons III, V and VI of the D. melanogaster verm may be alternatively spliced. Exons V and VI correspond to exons II and III in A. mellifera, arguing that they may be alternatively spliced, as well. By contrast, verm exon III does not show any similarity to any sequence in the $A$. mellifera genome. The sizes of introns are indicated between the exon boxes. b In D. melanogaster, A. mellifera, B. impatiens and $N$. vitripennis the genes coding Serp- and Verm-like chitin deacetylase are arranged in tandem. A full alignment of Serp, Verm and Serp/Verm-like proteins in D. melanogaster, A. mellifera and related insects is shown in Supplementary Figure 3.

shown to mediate partner recognition (Moussian et al 2005b). Taken together, like D. melanogaster Rtv, the A. mellifera orthologue is probably directed to the plasma membrane where it may interact with a partner to organise chitin microfibrils. The GB49764 transcript is detected at the larval and pupal stages (Table II).

\subsection{The chitin-binding factor obstructor-A}

Obstructor-A (Obst-A) is an extracellular chitin-binding protein that interacts with $\mathrm{Knk}$ and Serp to protect chitin from degradation in D. melanogaster (Petkau et al 2012). The ObstA protein has 237 residues constituting an $\mathrm{N}$ terminal signal peptide and three class 2 chitinbinding domains (Fig. S6). The A. mellifera potential orthologous sequence with 233 amino acids that is coded by the locus GB50636 (Figure 6) has the same domain composition. The proteins align over their entire length with $64.6 \%$ identical and $74.7 \%$ similar residues. The GB50636 protein shows significant similarity to a second $D$. melanogaster protein named obstructor-B that is highly similar to GB41227 (data not shown). GB50636 is $48.1 \%$ identical and $63.1 \%$ similar to obstructor-B (gaps $3.4 \%$ ). GB50636 is expressed at the larval and pupal stages (Table II).

\subsection{The transcription factor grainyhead}

The D. melanogaster grainyhead (grh) locus is complex. According to FlyBase, the grh gene has 19 exons coding for eight isoforms of a 


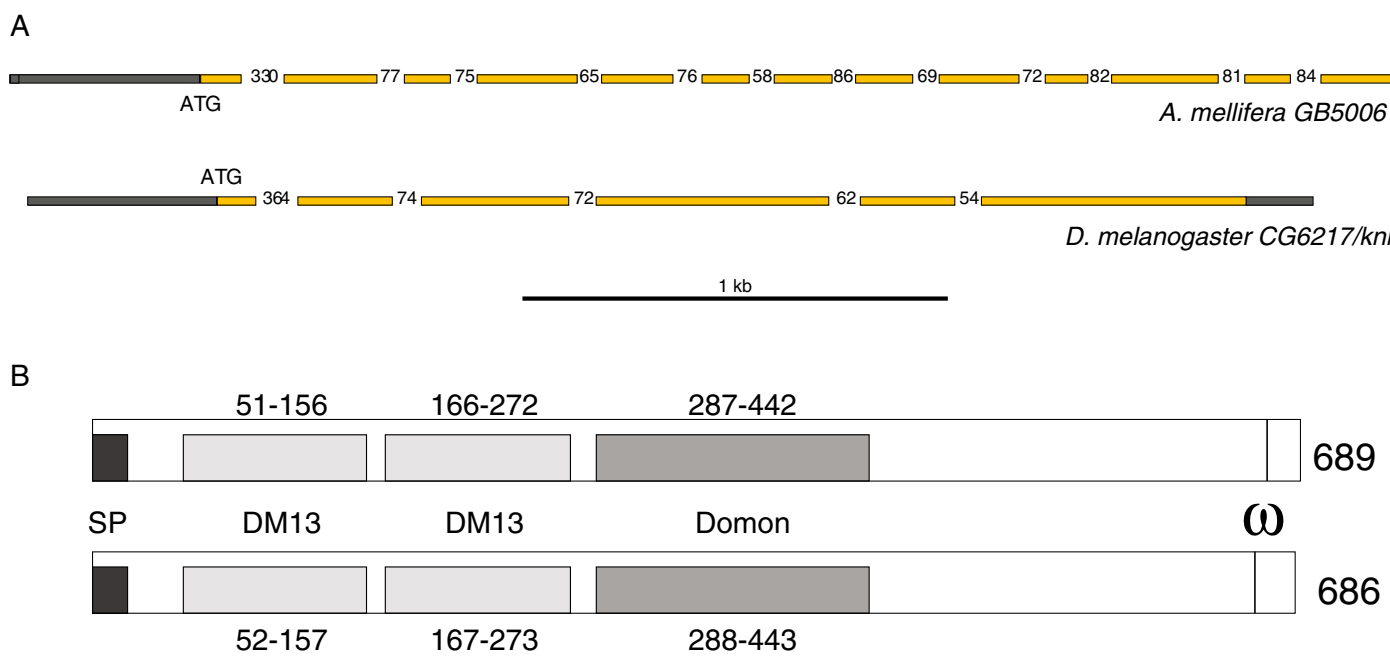

Figure 4. The knk locus. a The D. melanogaster knk gene has six exons (grey boxes represent non-coding sequences; orange boxes represent coding sequences) constituting a coding region of 2,067 bps. GB50061 has 13 exons adding up to 2,058 bps of coding sequence. b The resulting proteins align over 658 residues. Both proteins have an N-terminal signal peptide $(S P)$, followed by two DM13 domains (PF10517), a central DOMON domain (PF03351) and a C-terminal signature for GPI modification $(\omega)$. The DM13 domains between the proteins are 66 and $56 \%$ identical and 80.2 and $74.8 \%$ similar to each other, respectively. The DOMON domains share $64.1 \%$ identical and $78.8 \%$ similar amino acids. A full alignment of Knk and Knk-like proteins in D. melanogaster, A. mellifera and related insects is shown in Supplementary Figure 4. The sizes of introns are indicated between the exon boxes.

CP2-type transcription factor ranging from 155 to 1,333 residues (isoforms $\mathrm{PH}-\mathrm{O}$ ) that are needed for terminal epidermal and tracheal differentiation and in a subset of neurons in the D. melanogaster embryo (Bray and Kafatos
1991; Gangishetti et al 2012; Uv et al 1997). Experimentally, four isoforms $\left(\mathrm{N}, \mathrm{N}^{\prime}, \mathrm{O}\right.$ and $\left.\mathrm{O}^{\prime}\right)$ have been described coded by 17 exons (Uv et al 1997). The $\mathrm{N}$ isoforms (N 1,063 and $\mathrm{N}^{\prime} 1,032$ residues) are epidermal, while the $\mathrm{O}$ isoforms

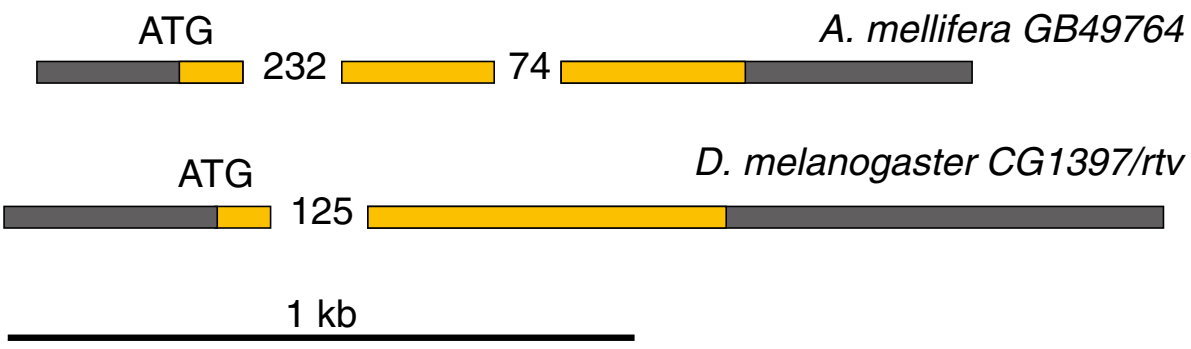

Figure 5. The $r t v$ locus. The $453 \mathrm{bps}$ open reading frame of the D. melanogaster rtv gene is split into two exons (grey boxes represent non-coding sequences; orange boxes represent coding sequences). GB49764 has three exons that together constitute an open reading frame of $438 \mathrm{bps}$. The position of the first intron is conserved separating the respective sequences coding for the signal peptide from the Ly6 domain (PF00021). A full alignment of Rtv and Rtv-like proteins in D. melanogaster, A. mellifera and related insects is shown in Supplementary Figure 5. The sizes of introns are indicated between the exon boxes. 
(O 1,333 and $\mathrm{O}^{\prime}$ 1,303 residues) are expressed in the nervous system. The grh homologous $A$. mellifera locus $G B 46725$ at BeeBase has 11 exons coding for a protein with 843 amino acids (Figure 7). The GB46725 transcript is detected at the larval and pupal stages (Table II). Whole transcriptome shotgun sequencing data at BeeBase indicate the additional existence of an alternative isoform with 636 amino acids. Alignment of the large $A$. mellifera protein with the $D$. melanogaster Grh epidermal isoform $\mathrm{N}$ reveals an identity of $61.4 \%$ and a similarity of $69.2 \%$ that is confined to the C-terminal half of the D. melanogaster protein (after around 600 aa, see Fig. S7). The Nterminal half of the D. melanogaster $\mathrm{N}$ and $\mathrm{O}$ isoforms is characterised by the presence of several stretches of multiple glutamines (12\%). The nonaligning N-terminal region of the $A$. mellifera Grhhomologous protein GB46725 is serine-rich $(15.5 \%)$. Consistently, the N-terminal half of the B. impatiens Grh-homologue Bimp10162 is serinerich, as well (Fig. S7). Because of the comparably shorter N-terminal region, it is possible that the GB46725 protein might have additional unmatchable and low-complex N-terminal sequences that have escaped annotation. Scanning of the 5 ' region as well as the large first and second introns of GB46725 for repetitive sequences failed to identify undetected exons coding for such sequences.

\section{DISCUSSION}

\subsection{Chitin synthesis and organisation in D. melanogaster and A. mellifera are generally conserved}

Our data reveal that genes coding for factors highly similar to those that are essential for chitin synthesis and organisation in D. melanogaster are present in $A$. mellifera. In other words, for each $D$. melanogaster gene, there is a counterpart in the $A$. mellifera genome. A central question is whether these factors are true orthologues or not. There are at least three criteria for true orthology. First, two factors in two species should have a common origin, i.e. they should be homologous. Second, the spatial and temporal expression patterns of these genes coding for homologous factors in two different species should be similar. Third, two homologous factors in two different species have to exert the same molecular and cellular function. Thus, the homologous factor from one species should be able to rescue the phenotype caused by a mutation in the respective gene in another species in transgenic experiments. Based on accurate sequence analyses, we present evidence that the chitin synthesis and organisation factors identified in $A$. mellifera are most probably orthologues of the respective factors, which have been genetically characterised in $D$. melanogaster. A strong argument that we have been comparing orthologous sequences is the determination of bidirectional best hits $(\mathrm{BBH})$, a simple method to infer orthology (Altenhoff and Dessimoz 2012): the most similar sequence retrieved by tBLASTx in A. mellifera, recognises the initial sequence of D. melanogaster as the most similar. In the case of $\mathrm{Knk}$, unambiguous reciprocal identification of similar sequences among three homologues in each species supports the assumption that GB650061 is the orthologue of Knk. Besides $\mathrm{BBH}$, we have additional arguments supporting the assumption that we have identified orthologues in the other cases.

Chitin synthase Insects have two genes coding for chitin synthases (Merzendorfer 2006). The defining trait of the epidermal chitin synthase versus the midgut version is an extended coiledcoil region including the WGTRE motif. Hence, the presence of this region indicates that GB49845 is the true orthologue of Kkv.

Serp and Verm Serp and Verm belong to the group I of insect chitin deacetlyses that harbours two isoforms per insect (Dixit et al 2008). They are characterised by an N-terminal chitinbinding domain, a middle LDL-like domain and a chitin deacetylase signature. In $D$. melanogaster, A. mellifera, B. impatiens and $N$. vitripennis, the respective genes are located in tandem in the genome. These features unambiguously support the assumption that the respective enzymes have a common origin and are true orthologues. 


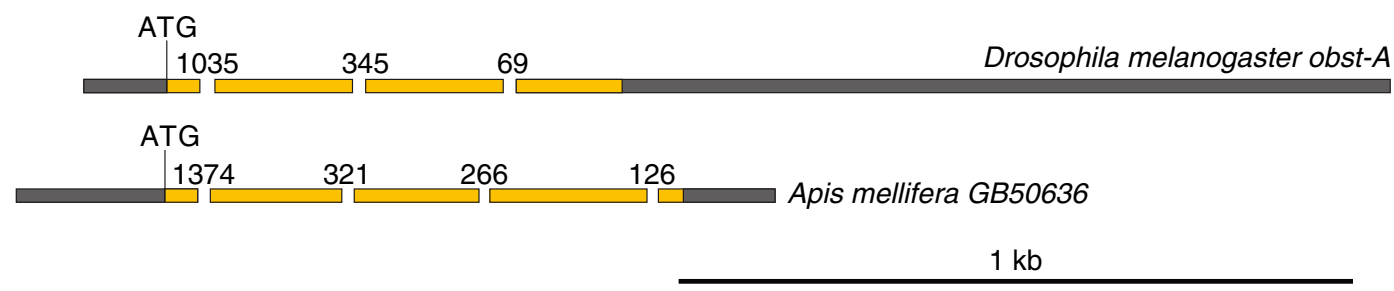

Figure 6. The obst-A locus. a The A. mellifera GB50636 locus that codes for an Obst-A homologous protein has five exons, whereas the D. melanogaster reference locus has four exons (grey boxes represent non-coding sequences; orange boxes represent coding sequences). A full alignment of Obst-A and Obst-A-like proteins in D. melanogaster, A. mellifera and related insects is shown in Supplementary Figure 6. The sizes of introns are indicated between the exon boxes.

Rtv and Mmy These two proteins are unique sequences in each insect. It is hence conceivable that GB44897 and GB49764 are orthologues of Rtv and Mmy, respectively.

Obst-A Similarly to Obst-B and Obst-B-like proteins, Obst-A and GB50636 have three class 2 chitin-binding domains. However, Obst-A and Obst-A-like proteins have a C-terminal ER retention signal suggesting that the cell biology between ObstA-proteins and Obst-B-proteins differs. In turn, this feature together with the BBH argument argues that Obst-A and GB50636 are orthologues.

Grh The A. mellifera and D. melanogaster genomes have been shown to code for two CP2type transcription factors (Huang et al 2012). Our
BLAST analyses revealed that GB46725 corresponds to Grh, whereas GB48238 is the cognate of Gemini. Grh and Grh-like proteins in the species examined have several yet uncharacterised regions C-terminal to the $\mathrm{CP} 2$ domain, which are completely missing in respective Gem proteins. Thus, despite N-terminal sequence divergences discussed below, the CP2type transcription factors described in this work are most probably Grh orthologues.

\subsection{Sequence divergences and expression differences}

Despite the identical set of chitin synthesis and organising factors in the fruit fly and the

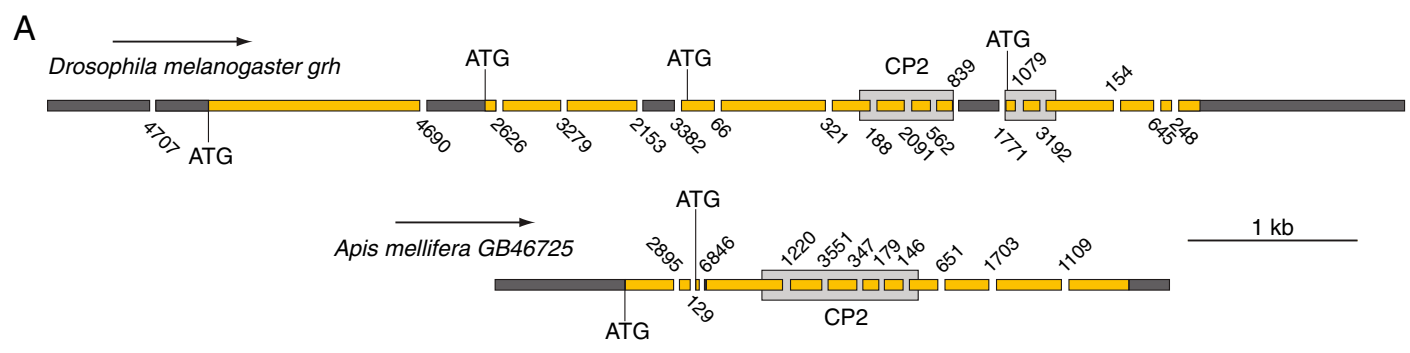

Figure 7. The grh locus. a According to the Flybase database, the D. melanogaster grh gene has 19 exons (dark grey boxes represent non-coding sequences; orange boxes represent coding sequences). The $A$. mellifera locus GB46725 is predicted to have 11 exons. To date, the D. melanogaster grh locus is annotated with four start codons $(A T G)$. The respective $A$. mellifera locus has only one start codon. b The CP2 domains (PF004516) of D. melanogaster (Dmel) and A. mellifera (Amel) are $85.8 \%$ identical and $90.7 \%$ similar to each other. A full alignment of Grh and Grh-like proteins in D. melanogaster, A. mellifera and related insects is shown in Supplementary Figure 7. The sizes of introns are indicated between the exon boxes. 
honeybee, differences exist in their sequence composition ( $k k v$ and $g r h)$ and expression pattern (serp and verm). Intriguingly, for instance, in $A$. mellifera and $B$. impatiens but not in other Hymenoptera tested, the sequence coding for the WGTRE motif of the epidermal chitin synthase is duplicated yielding two alternatively spliced exons. Of note, the WGTRE-coding sequence is also unique in other insect species belonging to orthopterans (Locusta), dipterans (e.g. Drosophila and Anopheles), coleopterans (e.g. Tribolium) and lepidopterans (e.g. Ostrinia) (Arakane et al 2004, 2005; Moussian et al 2005a; Qu and Yang 2011, 2012; Zhang et al 2010, 2012). Underlining the importance of this motif, a missense mutation changing the Gly to an Asp completely abrogates enzyme activity in D. melanogaster (Moussian et al 2005a). Moreover, the etoxazole sensitive isoleucine ${ }^{1056}$ (Van Leeuwen et al 2012) preceding the WGTRE motif is conserved in both alterative sequences. In summary, the alternative use of an important albeit molecularly uncharacterised motif in the A. mellifera and $B$. impatiens epidermal chitin synthases adds a level of complexity in chitin production that is absent in insects outside the Apoide clade.

Another major sequence difference in chitin synthesis and organising factors between $D$. melanogaster and A. mellifera concerns the Ntermini of the respective Grh proteins. The Nterminal half of epidermal Grh versions in $D$. melanogaster is glutamine-rich, a feature, which has been shown to enhance the activity of transcription factors (Atanesyan et al 2012). By contrast, the N-terminus of GB46725 in $A$. mellifera is serine-rich, a feature needed for transcription activation in Sp1-related transcription factors (Suske 1999). Considering the nonperfect alignment of N-termini between Grhlike proteins in $A$. mellifera-related species $B$. impatiens, C. floridanus and N. vitripennis, we conclude that in general, the $\mathrm{N}$-terminal region of insect Grh-like proteins is frequently modified during evolution.

Along with sequence divergences also discrepancies in gene expression profiles account for differences in chitin synthesis and organisation between $D$. melanogaster and A. mellifera beyond the basic scheme. Notably, the genes GB45151 and GB45152 coding for chitin deacetylases are expressed at different developmental stages in $A$. mellifera, whereas their orthologues serp and verm in D. melanogaster are expressed concomitantly at embryonic stages and act redundantly (Luschnig et al 2006). These genes have probably a common enhancer in D. melanogaster, whereas in $A$. mellifera, different enhancers control their expression at different developmental stages. This observation suggests a level of expression control in chitin organisation that is missing in D. melanogaster.

\subsection{Conclusion and outlook}

Recent publications on honeybee and fruit fly cuticle proteins underline considerable variances in cuticle composition in these two species. Whereas, for example, the $D$. melanogaster genome harbours 27 tweedle proteins, only two have been shown to be present in the A. mellifera genome (Guan et al 2006; Soares et al 2011). Moreover, sequences homologous to the three $A$. mellifera lowcomplex cuticle proteins called apidermins are missing in the D. melanogaster genome (Kucharski et al 2007). By contrast, in this study, we have shown that-despite some differences - the core of chitin synthesis and organisation machinery is most probably conserved between $D$. melanogaster and $A$. mellifera. Altogether, these findings underline that data from the fruit fly are only partially translatable to the honeybee and generally to other insects. Dissection of the molecular mechanisms of honeybee cuticle formation combined with a thorough histological study is hence crucial to contribute to the understanding of honeybee biology and ecology.

Our sequence analyses will serve to characterise essential chitin producing and organising factors in RNA interference experiments. Efforts focussing at pupal and pharate stages that have been histologically excellently described (Elias-Neto et al 2009) would be particularly rewarding. Ultimately, we expect to learn more about the interaction between honeybee 
and the pathogen vector Varroa destructor that transmits viruses through the cuticle (Rosenkranz et al 2010).

\section{Orthologues putatifs des gènes identifiés chez Drosophila melanogaster impliqués dans la synthese et l'organisation de la chitine chez Apis mellifera}

cuticule / chitine / synthase de la chitine

\begin{abstract}
Mutmaßliche Orthologe der bei Drosophila melanogaster identifizierten, zur Produktion und Organisation von Chitin benötigten gene bei Apis mellifera. Kutikula / Chitin / Chitin Synthase / Chitin-Organisation
\end{abstract}

\section{REFERENCES}

Altenhoff, A.M., Dessimoz, C. (2012) Inferring orthology and paralogy. Methods Mol Biol 855, 259-279

Arakane, Y., Hogenkamp, D.G., Zhu, Y.C., Kramer, K.J., Specht, C.A., Beeman, R.W., Kanost, M.R., Muthukrishnan, S. (2004) Characterization of two chitin synthase genes of the red flour beetle, Tribolium castaneum, and alternate exon usage in one of the genes during development. Insect Biochem Mol Biol 34, 291-304

Arakane, Y., Muthukrishnan, S., Kramer, K.J., Specht, C.A., Tomoyasu, Y., Lorenzen, M.D., Kanost, M., Beeman, R.W. (2005) The Tribolium chitin synthase genes TcCHS1 and TcCHS2 are specialized for synthesis of epidermal cuticle and midgut peritrophic matrix. Insect Mol Biol 14, 453-463

Araujo, S.J., Aslam, H., Tear, G., Casanova, J. (2005) mummy/cystic encodes an enzyme required for chitin and glycan synthesis, involved in trachea, embryonic cuticle and CNS development-analysis of its role in Drosophila tracheal morphogenesis. Dev Biol 288, 179-193

Atanesyan, L., Gunther, V., Dichtl, B., Georgiev, O., Schaffner, W. (2012) Polyglutamine tracts as modulators of transcriptional activation from yeast to mammals. Biol Chem 393, 63-70

Bray, S.J., Kafatos, F.C. (1991) Developmental function of Elf-1: an essential transcription factor during embryogenesis in Drosophila. Genes Dev 5, 1672-1683

Bustin, S.A., Benes, V., Garson, J.A., Hellemans, J., Huggett, J., Kubista, M., Mueller, R., Nolan, T., Pfaffl, M.W., Shipley, G.L., Vandesompele, J., Wittwer, C.T. (2009) The MIQE guidelines: minimum information for publication of quantitative real-time PCR experiments. Clin Chem 55, 611-622
Chaudhari, S.S., Arakane, Y., Specht, C.A., Moussian, B., Boyle, D.L., Park, Y., Kramer, K.J., Beeman, R.W., Muthukrishnan, S. (2011) Knickkopf protein protects and organizes chitin in the newly synthesized insect exoskeleton. Proc Natl Acad Sci U S A 108, 17028-17033

Chaudhari, S.S., Arakane, Y., Specht, C.A., Moussian, B., Kramer, K.J., Muthukrishnan, S., Beeman, R.W. (2013) Retroactive maintains cuticle integrity by promoting the trafficking of Knickkopf into the procuticle of Tribolium castaneum. PLoS Genet 9, e1003268

Dixit, R., Arakane, Y., Specht, C.A., Richard, C., Kramer, K.J., Beeman, R.W., Muthukrishnan, S. (2008) Domain organization and phylogenetic analysis of proteins from the chitin deacetylase gene family of Tribolium castaneum and three other species of insects. Insect Biochem Mol Biol 38, 440-451

Elias-Neto, M., Soares, M.P., Bitondi, M.M. (2009) Changes in integument structure during the imaginal molt of the honey bee. Apidologie 40, 29-39

Gangishetti, U., Veerkamp, J., Bezdan, D., Schwarz, H., Lohmann, I., Moussian, B. (2012) The transcription factor Grainy head and the steroid hormone ecdysone cooperate during differentiation of the skin of Drosophila melanogaster. Insect Mol Biol 21, 283295

Guan, X., Middlebrooks, B.W., Alexander, S., Wasserman, S.A. (2006) Mutation of TweedleD, a member of an unconventional cuticle protein family, alters body shape in Drosophila. Proc Natl Acad Sci U S A 103, 16794-16799

Hogenkamp, D.G., Arakane, Y., Zimoch, L., Merzendorfer, H., Kramer, K.J., Beeman, R.W., Kanost, M.R., Specht, C.A., Muthukrishnan, S. (2005) Chitin synthase genes in Manduca sexta: characterization of a gut-specific transcript and differential tissue expression of alternately spliced mRNAs during development. Insect Biochem Mol Biol 35, 529-540

Huang, L., Cheng, T., Xu, P., Fang, T., Xia, Q. (2012) Bombyx mori transcription factors: genome-wide identification, expression profiles and response to pathogens by microarray analysis. J Insect Sci 12, 40

Irion, U. (2012) Drosophila muscleblind codes for proteins with one and two tandem zinc finger motifs. PLoS One 7, e34248

Johnson, B.R., Borowiec, M.L., Chiu, J.C., Lee, E.K., Atallah, J., Ward, P.S. (2013) Phylogenomics resolves evolutionary relationships among ants, bees, and wasps. Curr Biol 23, 2058-2062

Kucharski, R., Maleszka, J., Maleszka, R. (2007) Novel cuticular proteins revealed by the honey bee genome. Insect Biochem Mol Biol 37, 128-134

Locke, M. (2001) The Wigglesworth Lecture: Insects for studying fundamental problems in biology. J Insect Physiol 47, 495-507

Luschnig, S., Batz, T., Armbruster, K., Krasnow, M.A. (2006) serpentine and vermiform encode matrix 
proteins with chitin binding and deacetylation domains that limit tracheal tube length in Drosophila. Curr Biol 16, 186-194

Merzendorfer, H. (2006) Insect chitin synthases: a review. J Comp Physiol B 176, 1-15

Moussian, B. (2008) The role of GlcNAc in formation and function of extracellular matrices. Comp Biochem Physiol B Biochem Mol Biol 149, 215-226

Moussian, B. (2010) Recent advances in understanding mechanisms of insect cuticle differentiation. Insect Biochem Mol Biol 40, 363-375

Moussian, B. (2013) The apical plasma membrane of chitinsynthesizing epithelia. Insect science 20, 139-146

Moussian, B., Schwarz, H., Bartoszewski, S., NussleinVolhard, C. (2005a) Involvement of chitin in exoskeleton morphogenesis in Drosophila melanogaster. J Morphol 264, 117-130

Moussian, B., Soding, J., Schwarz, H., NussleinVolhard, C. (2005b) Retroactive, a membraneanchored extracellular protein related to vertebrate snake neurotoxin-like proteins, is required for cuticle organization in the larva of Drosophila melanogaster. Dev Dyn 233, 1056-1063

Moussian, B., Tang, E., Tonning, A., Helms, S., Schwarz, H., Nusslein-Volhard, C., Uv, A.E. (2006) Drosophila Knickkopf and Retroactive are needed for epithelial tube growth and cuticle differentiation through their specific requirement for chitin filament organization. Development 133, 163-171

Munoz-Torres, M.C., Reese, J.T., Childers, C.P., Bennett, A.K., Sundaram, J.P., Childs, K.L., Anzola, J.M., Milshina, N., Elsik, C.G. (2011) Hymenoptera Genome Database: integrated community resources for insect species of the order Hymenoptera. Nucleic Acids Res 39, D658-662

Pare, A., Kim, M., Juarez, M.T., Brody, S., McGinnis, W. (2012) The functions of grainy head-like proteins in animals and fungi and the evolution of apical extracellular barriers. PLoS One 7, e36254

Petkau, G., Wingen, C., Jussen, L.C., Radtke, T., Behr, M. (2012) Obstructor-A is required for epithelial extracellular matrix dynamics, exoskeleton function, and tubulogenesis. J Biol Chem 287, 21396-21405

Qu, M., Yang, Q. (2011) A novel alternative splicing site of class A chitin synthase from the insect Ostrinia furnacalis - gene organization, expression pattern and physiological significance. Insect Biochem Mol Biol 41, 923-931

Qu, M., Yang, Q. (2012) Physiological significance of alternatively spliced exon combinations of the single-copy gene class A chitin synthase in the insect Ostrinia furnacalis (Lepidoptera). Insect Mol Biol 21, 395-404
Rezende, G.L., Martins, A.J., Gentile, C., Farnesi, L.C., Pelajo-Machado, M., Peixoto, A.A., Valle, D. (2008) Embryonic desiccation resistance in Aedes aegypti: presumptive role of the chitinized serosal cuticle. BMC Dev Biol 8, 82

Rosenkranz, P., Aumeier, P., Ziegelmann, B. (2010) Biology and control of Varroa destructor. J Invertebr Pathol 103(Suppl 1), S96-119

Schimmelpfeng, K., Strunk, M., Stork, T., Klambt, C. (2006) Mummy encodes an UDP-Nacetylglucosamine-dipohosphorylase and is required during Drosophila dorsal closure and nervous system development. Mech Dev 123, 487-499

Soares, M.P., Silva-Torres, F.A., Elias-Neto, M., Nunes, F.M., Simoes, Z.L., Bitondi, M.M. (2011) Ecdysteroid-dependent expression of the tweedle and peroxidase genes during adult cuticle formation in the honey bee, Apis mellifera. PLoS One 6, e20513

Soding, J., Biegert, A., Lupas, A.N. (2005) The HHpred interactive server for protein homology detection and structure prediction. Nucleic Acids Res 33, W244-248

Suske, G. (1999) The Sp-family of transcription factors. Gene 238, 291-300

Tonning, A., Helms, S., Schwarz, H., Uv, A.E., Moussian, B. (2006) Hormonal regulation of mummy is needed for apical extracellular matrix formation and epithelial morphogenesis in Drosophila. Development 133, 331-341

Uv, A.E., Harrison, E.J., Bray, S.J. (1997) Tissuespecific splicing and functions of the Drosophila transcription factor Grainyhead. Mol Cell Biol 17, 6727-6735

Van Leeuwen, T., Demaeght, P., Osborne, E.J., Dermauw, W., Gohlke, S., Nauen, R., Grbic, M., Tirry, L., Merzendorfer, H., Clark, R.M. (2012) Population bulk segregant mapping uncovers resistance mutations and the mode of action of a chitin synthesis inhibitor in arthropods. Proc Natl Acad Sci U S A 109, 44074412

Zhang, J., Liu, X., Zhang, J., Li, D., Sun, Y., Guo, Y., Ma, E., Zhu, K.Y. (2010) Silencing of two alternative splicing-derived mRNA variants of chitin synthase 1 gene by RNAi is lethal to the oriental migratory locust, Locusta migratoria manilensis (Meyen). Insect Biochem Mol Biol 40, 824-833

Zhang, X., Zhang, J., Park, Y., Zhu, K.Y. (2012) Identification and characterization of two chitin synthase genes in African malaria mosquito, Anopheles gambiae. Insect Biochem Mol Biol 42, 674-682 